\title{
Vectorial Transcellular Calcium Transport in Intestine: Integration of Current Models
}

\author{
Dennis Larsson ${ }^{1,2}$ and Ilka Nemere ${ }^{1 *}$ \\ ${ }^{1}$ Department of Nutrition and Food Sciences and the Biotechnology Center, Utah State University, Logan, UT 85322-8700, USA \\ ${ }^{2}$ Fish Endocrinology Laboratory, Department of Zoology/Zoophysiology, \\ Göteborg University, Box 463, SE-405 30 Göteborg, Sweden
}

Received 19 March 2002; accepted 29 April 2002

\begin{abstract}
In spite of decades of research, the exact subcellular pathway for calcium transport in intestine has not been elucidated. In this mini-review, we present three models for vectorial movement of calcium across the cell: facilitated (cytoplasmic) diffusion, vesicular/lysosomal transport, and tunneling through the endoplasmic reticulum compartment. We conclude by offering one way to integrate elements of these three models.
\end{abstract}

\section{INTRODUCTION}

While a number of models have been proposed to explain the transport of calcium across polarized epithelium such as that found in the intestine, each potential mechanism also relies on some points of speculation. Many of these models have been developed within the context of the vitamin $\mathrm{D}$ endocrine system in which a hormonally active metabolite, 1,25-dihdyroxyvitamin $\mathrm{D}_{3}$, promotes the transport. Thus, numerous observations compare cellular and molecular events as a function of time after repletion of vitamin D-deficient animals with the seco-steroid hormone. In this manner, Wasserman and Taylor [1] reported the vitamin D-induced calbindins, and Nemere (see [2], for review) found that vesicular carriers may be viable candidates for calcium transporters. In this mini-review, we present the models as they where chronologically proposed, as well as one that has not yet been evaluated in the intestine. We conclude by suggesting points where elements of these models can be integrated.

\section{INTESTINAL CALCIUM TRANSPORT}

Intestinal calcium transport can be described as a threestep process: calcium entry across the apical membrane, transcellular transport, and extrusion across the basolateral membrane. Three models for intestinal calcium transport have been postulated: (1) facilitated diffusion, (2) vesicular transport, and (3) tunneling through intracellular stores (endoplasmic reticulum).

\section{Facilitated diffusion}

In this model, calcium enters the intestinal cell through calcium channels in the apical membrane [3]. Upon entry, calcium binds to calbindins (calcium binding proteins induced by the steroid hormone 1,25-dihydroxyvitamin $\mathrm{D}_{3}$ ), which transport calcium through the cytoplasm to the basolateral membrane. At the basolateral membrane calcium is transferred from the calbindin to a higher affinity Ca-ATPase or $\mathrm{Na} / \mathrm{Ca}$-exchanger, which in turn transports the divalent cation to the extracellular media $[4,5]$.

\section{Vesicular transport}

Formation of calcium-enriched vesicles is initiated by influx of the cation through calcium channels in the apical membrane. The rapid increase in calcium concentrations close to the apical membrane, disrupts the actin filaments near the calcium channels and initiates the formation of endocytic vesicles. Concurrently, calcium ions bind to calmodulin (CaM) associated with myosin I or alternatively CaM associated with the calcium channels, which leads to an inactivation of the channels. This inactivation of the calcium channels causes a decrease in the free calcium levels close to the apical membrane and the actin filament network can be restored. Alternatively, influx through a calcium channel may promote the exocytotic delivery of vesicles containing calcium transporters $[4,6]$, which then are coupled to the formation of endocytic vesicles. The newly formed calciumcontaining vesicle is transported by microtubules and some fuse with lysosomes [2]. Both the transport vesicles and lysosomes have been reported to contain high levels of calbindin [7]. Ultimately, the vesicles/lysosomes are delivered to the basolateral membrane where they fuse with the membrane and the calcium content is extruded to the extracellular media. Fusion of vesicles with the basolateral membrane is dependent on calcium influx through calcium channels, which disrupts the actin filaments close to the membrane $[2,4]$. 


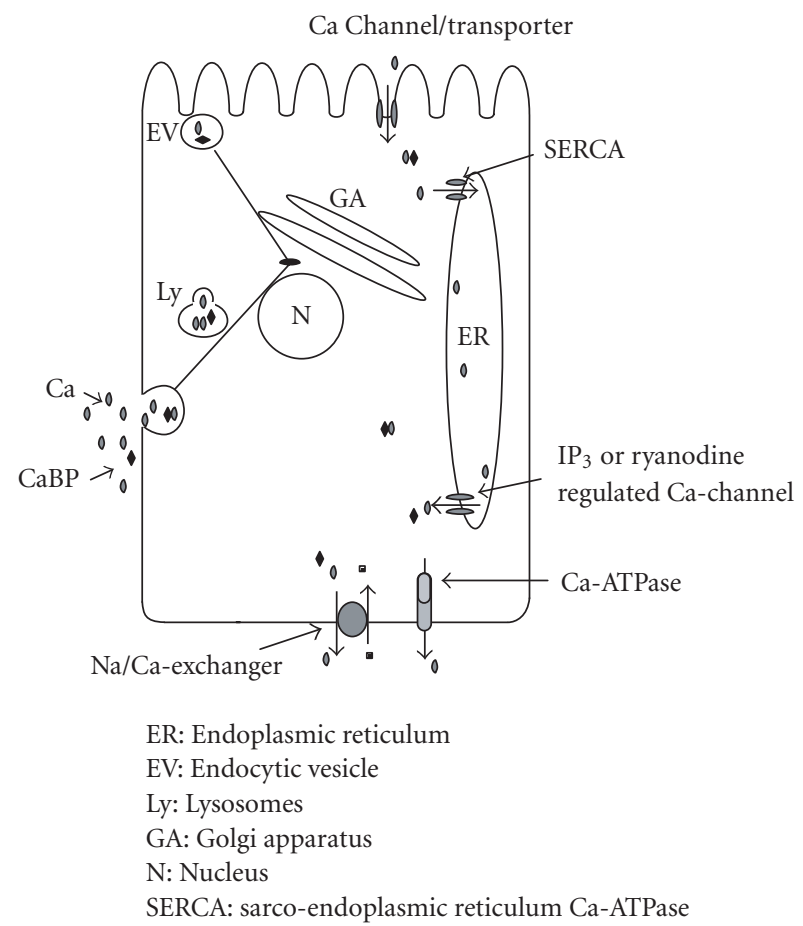

Figure 1. Vectorial transcellular calcium transport in intestinal epithelium. Three distinct but potentially complementary pathways are depicted: (1) uptake and transport of calcium (and calbindin) by vesicles/lysosomes with transport along microtubules, and fusion with the basolateral membrane to complete delivery of the cation; (2) entry of calcium into the cytoplasm with binding to calbindin and diffusion to the basal lateral membrane for extrusion by Ca-ATPase; (3) entry of calcium into the cytoplasm and uptake by the ER, diffusion through the ER, and extrusion of calcium in the vicinity of Ca-ATPases and $\mathrm{Na} / \mathrm{Ca}$-exchangers.

\section{Tunneling through intracellular stores}

This model has been demonstrated in pancreatic acinar cells [8], and constitutes a possible calcium transport route in enterocytes. As in the previous model of facilitated diffusion, calcium enters the intestinal cell through calcium channels. The transport from the apical to the basolateral side occurs through passive diffusion in the endoplasmic reticulum (ER). This transport pathway involves active buffering of calcium rather than passive buffering as in the case of facilitated diffusion. Calcium enters the sarco-endoplasmic reticulum via a Ca-ATPase (SERCA) and is released at the basolateral membrane through calcium release channels; the latter activity is regulated by $\mathrm{IP}_{3}$ and ryanodine receptors. Calcium extrusion to the extracellular media is governed by Ca-ATPases and $\mathrm{Na} / \mathrm{Ca}$-exchangers in the basolateral membrane.

\section{INTEGRATION OF THE THREE MODELS}

The intestine plays an important role in vertebrate calcium regulation. Depending on the activity and the demand of the animal, intestinal calcium uptake has to be regulated on a short-term and a long-term basis. This regulation is governed by endocrine factors such as $\mathrm{PTH}, 1,25(\mathrm{OH})_{2} \mathrm{D}_{3}$, and $24,25(\mathrm{OH})_{2} \mathrm{D}_{3}$. Short-term regulation includes activity of calcium transporters and channels together with formation of transport vesicles. Long-term regulation involves synthesis of key proteins for the cellular transport pathway, to increase calcium movement and buffering capacity.

Vesicular calcium transport and tunneling calcium through the ER are mechanisms that are important in the short-term regulation of intestinal calcium transport. Both transport pathways can be activated within seconds to minutes by endocrine factors. Cytoplasmic transport of calcium by calbindin is the slower mechanism, and the effects on intestinal calcium transport via this pathway are observed hours after hormone stimulation. However, calbindins and the ER are important in the short term for buffering cytoplasmic calcium concentrations. Figure 1 describes an integrated model for intestinal calcium transport.

\section{CONCLUDING REMARKS}

It is conceivable that calcium transport across polarized epithelia encompasses elements of all three models. "Signalling" calcium from the apical membrane may tunnel through ER compartments to contribute to the net transported ions. Vesicular calcium, which is capable of vectorial transport along microtubules, might also provide a store of "signalling" [9] as well as transport calcium. Finally, calcium transported by the facilitated diffusion model might be responsible for maintaining the supply of cation after the rapid delivery mechanisms have ceased.

\section{ACKNOWLEDGMENTS}

This work was supported by the Utah Agricultural Experiment Station. Approved as journal paper no. 7461. Also supported by the Swedish Foundation for International Cooperation in Research and Higher Education 99/820(DL).

\section{REFERENCES}

[1] Wasserman RH, Taylor AM. Vitamin $\mathrm{D}_{3}$-induced calcium-binding protein in chick intestinal mucosa. Science. 1966;152:791-793.

[2] Nemere I. Genomic and nongenomic actions of vitamin D on calcium transport in intestine. Poultry and Avian Biology Reviews. 1996;7:205-216.

[3] Larsson D, Nemere I. 24,25-dihydroxyvitamin $\mathrm{D}_{3}$ and intestinal calcium transport: from receptors to physiological response. In: Pandalai SG, ed. Recent research developments in endocrinology. vol. 2(1). Trivandrum, India: Transworld research network; 2001:183-198.

[4] Wasserman RH, Chandler JS, Meyer SA, et al. Intestinal calcium transport and calcium extrusion processes at the basolateral membrane. J Nutr. 1992;122(Suppl 3):662671.

[5] Slepchenko BM, Bronner F. Modeling of transcellular Ca transport in rat duodenum points to coexistence of two mechanisms of apical entry. Am J Physiol Cell Physiol. 2001;281(1):C270-C281. 
[6] Smith RM, Baibakov B, Ikebuchi Y, et al. Exocytotic insertion of calcium channels constrains compensatory endocytosis to sites of exocytosis. J Cell Biol. 2000;148(4):755-767.

[7] Nemere I, Leathers VL, Thompson BS, Luben RA, Norman AW. Redistribution of calbindin- $\mathrm{D}_{28 k}$ in chick intestine in response to calcium transport. Endocrinology. 1991;129(6):2972-2984.

[8] Petersen OH, Fedirko NV. Calcium signalling: store-operated channel found at last. Curr Biol. 2001;11(13):R520-R523.

[9] Haller T, Dietl P, Deetjen P, Volkl H. The lysosomal compartment as intracellular calcium store in MDCK cells: a possible involvement in $\mathrm{InsP}_{3}$-mediated $\mathrm{Ca}^{2+}$ release. Cell Calcium. 1996;19(2):157-165.

* Corresponding author.

E-mail: nemere@cc.usu.edu

Fax: +1 435797 2379; Tel: +1 4357973286 

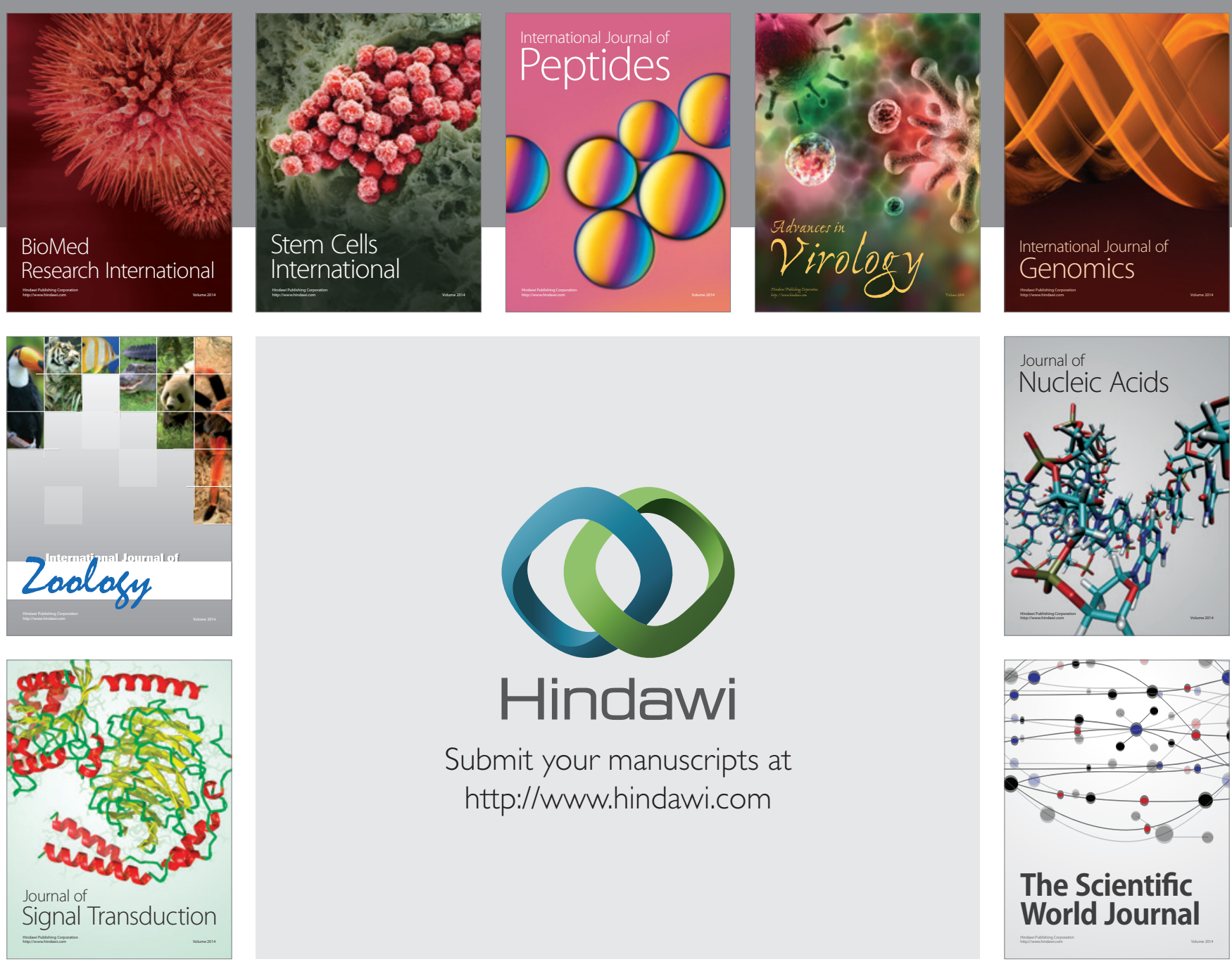

Submit your manuscripts at

http://www.hindawi.com
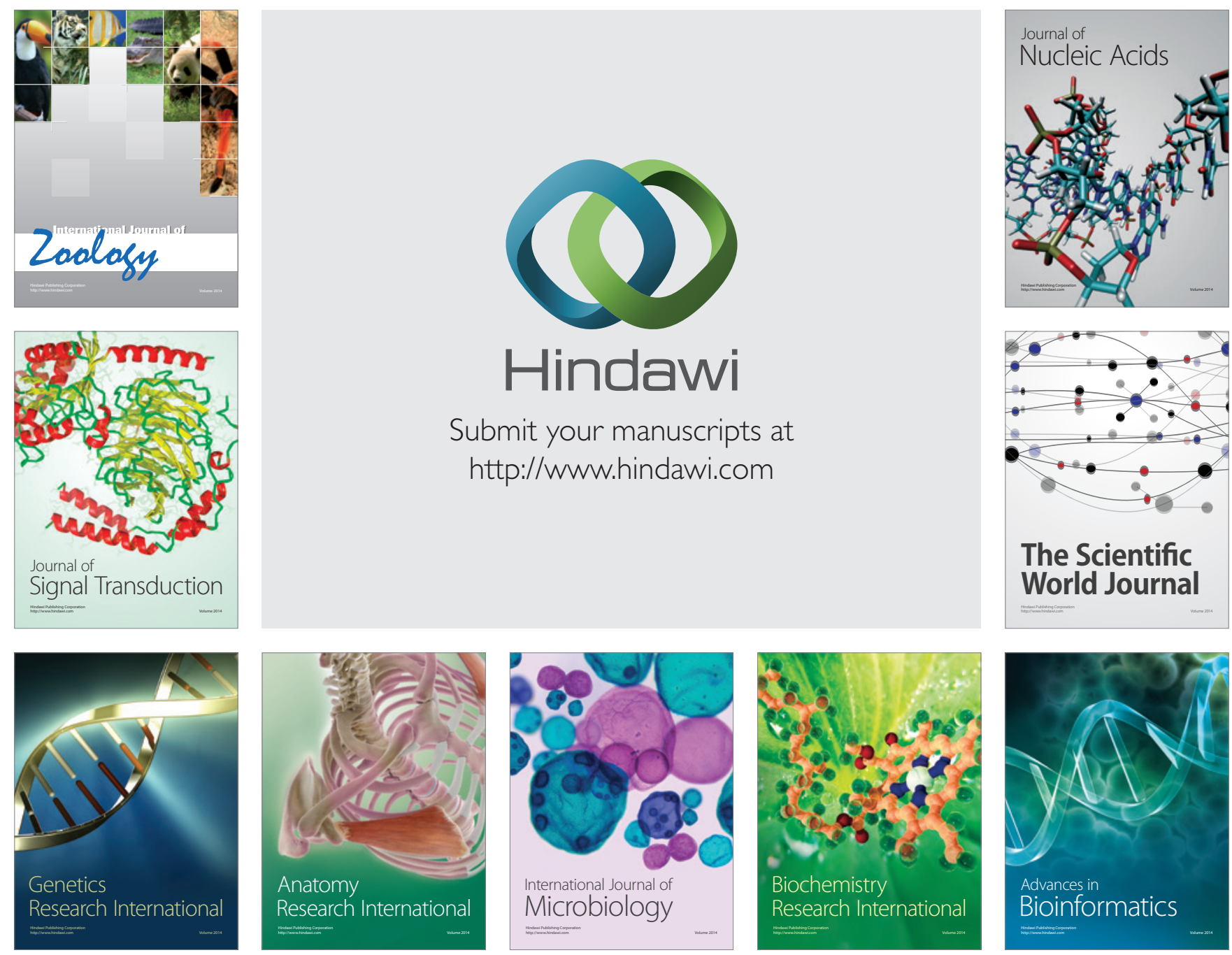

The Scientific World Journal
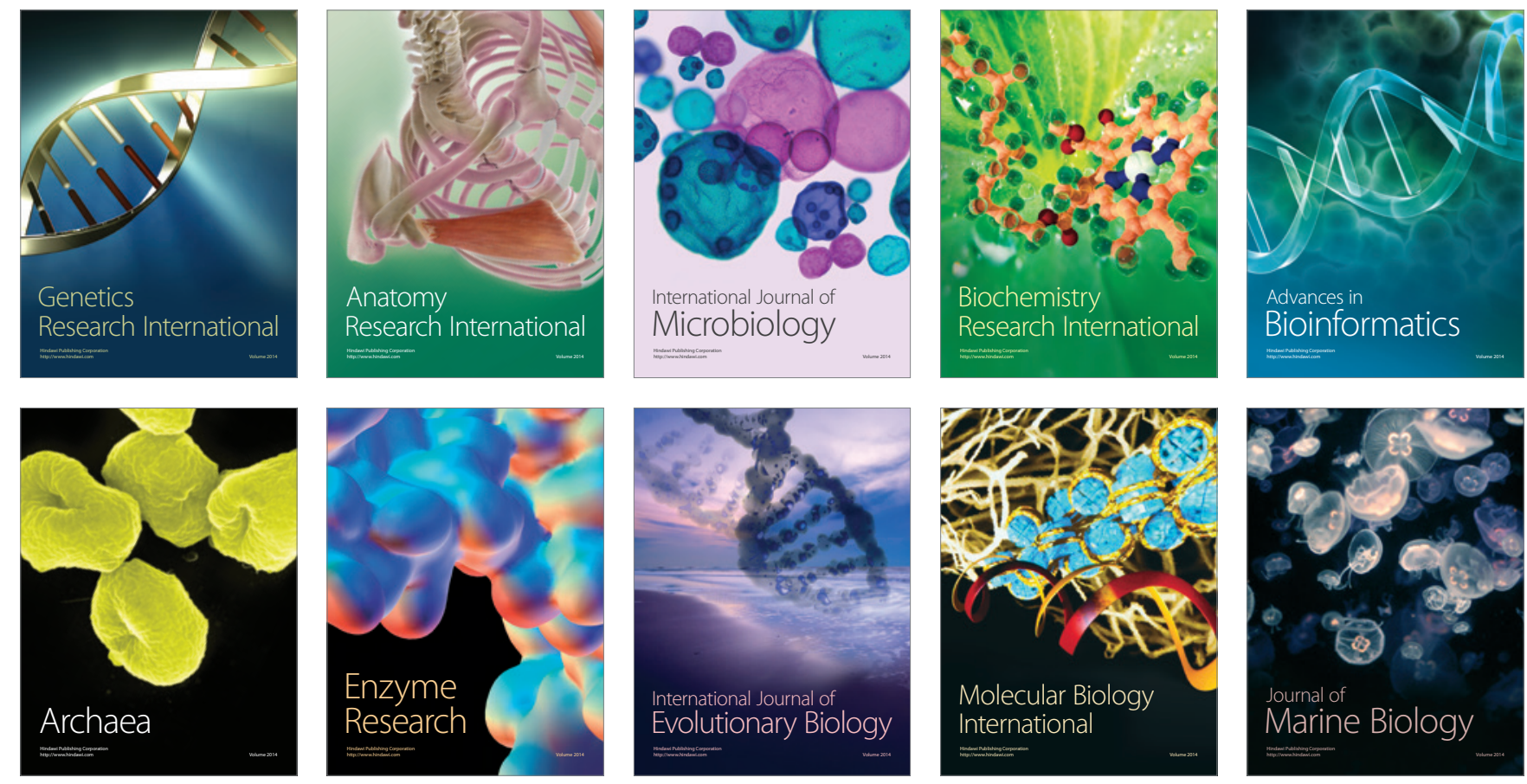